\title{
COVID-19 У ПАЦИЕНТОВ С ВИЧ-ИНФЕКЦИЕЙ
}

(ОБЗОР ЛИТЕРАТУРЫ).

\section{Долгова Н.Н., Рындич А.А., Твердохлебова Т.И., Суладзе А.Г. \\ ФБУН «Ростовский научно-исследовательский институт микробиологии и паразитологии» Роспотребнадзора, г. Ростов-на-Дону}

\section{Резюме:}

Цель работы: изучение особенностей клинического течения новой коронавирусной инфекции у людей живущих с ВИЧ и некоторые аспекты их лечения.

Представлен анализ научных публикаций по вопросу клиникоэпидемиологических аспектов COVID-19 у больных ВИЧ-инфекцией. На основе имеющихся литературных данных проведена оценка наличия ВИЧинфекции, как предиктора тяжелого течения и высокого процента летальности от COVID-19. Людям с ВИЧ показано следовать стандартным мерам профилактики новой коронавирусной инфекции.

Ключевые слова: COVID-19, SARS-CoV-2, ВИЧ-инфекция, антиретровирусные препараты, ЛЖВ.

\section{Введение:}

В декабре 2019 г. в китайском в городе Ухань (Wuhan City), столице провинции Хубэй (Hubei Province of China), стали регистрироваться случаи пневмонии неясной этиологии. Вскоре было обнаружено, что возбудитель относится к семейству коронавирусов, но не принадлежит ни к одному известному варианту. Новое заболевание получило временно утвержденное название - новая коронавирусная инфекция (2019-nCoV). Уже к 3 января 2020 г. диагноз пневмония, вызванная новым коронавирусом, был поставлен 44 пациентам [1-2]. Вирус быстро преодолел границы Китая и стал массово распространяться по всему миру. К 31 января 2020 г. первые случаи инфекции 
были зафиксированы в России. Вскоре инфекция получила название COVID19, а сам вирус стал обозначаться, как SARS-CoV-2.

11 марта 2020 года ВОЗ объявила о начале пандемии новой коронавирусной инфекции. В Ростовской области первый случай выявления SARS-CoV-2 был зарегистрирован 25 марта 2020 года у женщины, прибывшей из стран дальнего зарубежья незадолго до проявления симптомов заболевания. С этого момента эпидемия новой коронавирусной инфекции стала быстро распространяться по территории Ростовской области.

Особенностью, привлекшей внимание специалистов всех сфер здравоохранения и вызвавшей всеобщие опасения, стал факт преодоления вирусом предела уровня допустимого биологического риска. Ранее такой особенности среди представителей этого семейства, как правило, не наблюдалось [3]. Однако стоит упомянуть эпидемические вспышки коронавирусов SARS-CoV (2002 г.) и MERS-CoV (2012 г.), которые также доказывают способность этой группы возбудителей вызывать тяжелые инфекционные процессы у человека. В настоящее время известно о циркуляции среди населения четырех коронавирусов (HCoV-229E, -OC43, NL63 и -HKU1), которые круглогодично присутствуют в структуре ОРВИ, и, как правило, вызывают поражение верхних дыхательных путей легкой и средней степени тяжести.

Говоря о благополучии населения в условиях эпидемического процесса, который охватывает практически всю территорию земного шара, в первую очередь необходимо вспомнить об уязвимых в отношении к новой инфекции группах, в одну из которых могут входить лица живущие с ВИЧ (далее ЛЖВ).

Исходя из вышесказанного, в настоящее время главными вопросами в борьбе с новой коронавирусной инфекцией является точное определение рисков течения заболевания среди особых групп пациентов, а также поиск эффективной этиотропной терапии для всех категорий граждан. Отдельное 
место в этих вопросах занимают ЛЖВ, опасения о повышенном риске заражения которых, имелись еще до объявления ВОЗ о начале пандемии COVID-19.

Исследуя проблему эпидемии новой коронавирусной инфекции среди популяции людей с ВИЧ, стоит брать во внимание, что одним из первых препаратов для лечения COVID-19 во Временных методических рекомендациях "Профилактика, диагностика и лечение новой коронавирусной инфекции" от 3 февраля 2020 г., утвержденных Министерством здравоохранения Российской Федерации выступил антиретровирусный препарат в фиксированной комбинации доз лопинавир/ритонавир (торговое название «Калетра»). В седьмой редакции документа от 03.06.2020 г. также рекомендуется проводить этиотропное лечение данным препаратом. «Калетра», достаточно длительное время применяется для лечения ВИЧинфекции. На фоне этих данных учеными по всему миру проводятся исследования особенностей заражения COVID-19, характера и тяжести течения заболевания у ЛЖВ. Во многих источниках оговаривается предположение о том, что препарат «Калетра», наряду с эффективным действием против ВИЧ-инфекции способен блокировать и некоторые ферменты SARS-CoV-2. В связи с этим, рассматривается вопрос о взаимосвязи приема антиретровирусных препаратов и тяжести течения COVID-19. В некоторых источниках выдвинуто предположение о том, что прием этих лекарственных средств наряду с подавлением экспрессии ВИЧ, также способен препятствовать репликации SARS-CoV-2 в организме, что способствует более легкому течению COVID-19. Но в настоящее время нет актуальных исследований, подтверждающих эту теорию.

Первое рандомизированное клиническое исследование с использованием лопинавира/ритонавира началось через 13 дней после появления симптомов и не показало преимуществ по сравнению со стандартным лечением у 199 взрослых, госпитализированных с тяжелой формой COVID-19 [4]. Нет данных 
о том, что данную комбинацию препаратов применяли у тяжелых больных, так как это лекарственное средство затруднительно давать пациентам, подключенным к аппаратам искусственной вентиляции легких, и также отсутствует информация, подтверждающая успешное использование других антиретровирусных препаратов в лечении новой коронавирусной инфекции. Также было высказано предположение о том, что концентрация лопинавира/ритонавира, которая достигается и поддерживается при терапии ВИЧ-инфекции, является слишком низкой для подавления репликации вируса SARS-CoV-2.

При прогнозировании возможности развития крайне тяжелого течения заболевания и неблагоприятного исхода инфекции COVID-19 у пациентов с ВИЧ, в первую очередь, необходимо принимать во внимание стойкую иммуносупрессию (низкое количество CD4 лимфоцитов) и отсутствие проводимого антиретровирусного лечения. Однако пока мы не имеем данных о таких пациентах. Но, не стоит забывать и о том, что введение карантинных мер и других ограничительных мероприятий негативно отразилось на оказании помощи ВИЧ-инфицированным. Для граждан был ограничен доступ к тестированию на ВИЧ. Помимо этого, во время строгого соблюдения наложенных в связи с пандемией COVID-19 ограничений возникли затруднения со своевременным назначением антиретровирусной терапии, включая корректировку терапевтической схемы, поскольку врачиинфекционисты, специализирующиеся на лечении ВИЧ, были вынуждены выполнять обязанности по диагностике и лечению COVID-19. Данные, свидетельствующие этому, были продемонстрированы в более чем 50\% клиник в Центральной и Восточной Европе [5].

Во Временных методических рекомендациях "Профилактика, диагностика и лечение новой коронавирусной инфекции (COVID-19)" выделены особые группы пациентов: 
- больные с артериальной гипертензией;

- больные с гиперлипидемией;

- больные с острым коронарным синдромом;

- больные сахарным диабетом;

- больные с хронической обструктивной болезнью легких;

- больные с интерстициальными, редкими и генетически детерминированными заболеваниями легких;

- больные с иммуновоспалительными ревматическими заболеваниями.

В этот перечень не входят ВИЧ-инфицированные. Несмотря на то, что люди с ВИЧ находящиеся на лечении с нормальным количеством CD4 лимфоцитов и неопределяемой вирусной нагрузкой, могут не подвергаться повышенному риску серьезных заболеваний, многие ЛЖВ имеют другие состояния, которые повышают их риск [6]. Описана серия случаев обнаружения COVID-19 у ВИЧ-инфицированных в Китае, Испании, Германии, Италии и США.

В частности, группой немецких авторов была издана статья, описывающая исследование, проведенное среди 12 СПИД-центров на территории Германии, в которых был проведен ретроспективный анализ случаев течения COVID-19 у 33 пациентов с ВИЧ-инфекцией. Средний возраст пациентов составлял 48 лет (диапазон 26-82 года). Во всех анализируемых случаях у ЛЖВ методом ПЦР было подтверждено наличие SARS-CoV-2. Практически у всех (30 человек) наблюдалась низкая вирусная нагрузка, все пациенты находились на антиретровирусной терапии. Из общего числа анализируемых случаев, $60 \%$ госпитализированных имели сопутствующие патологии, определяемые как факторы риска развития тяжелого течения COVID-19 (артериальная гипертензия, сахарный диабет, сердечно-сосудистые заболевания, хроническая обструктивная болезнь легких, хроническая почечная недостаточность). 
Наиболее частыми симптомами были кашель (78\%), лихорадка (69\%), артралгия и миалгия (22\%), головная боль (22\%), боль в горле (22\%). Потеря вкуса и обоняния наблюдалась у 19\% пациентов. Тяжесть течения заболевания распределилась следующим образом: среднетяжелая форма - 76\%, тяжелая форма - 6\%, а 18\% больных находились в крайне тяжелой форме. Тогда как большая часть случаев завершилась выздоровлением (91\%), летальный исход имел место в 9\% случаев (у всех умерших в анамнезе присутствовали факторы риска тяжелого течения COVID-19). Клинические характеристики COVID-19 среди ЛЖВ, по-видимому, не отличаются от таковых в общей популяции. Кроме того, все пациенты получали антиретровирусную терапию, и все, кроме четырех, имели CD4 + T-клетки > 350/Mм3, что указывает на отсутствие серьезного иммунодефицита.

Авторами отмечено, что только двум пациентам с обнаруживаемой виремией, требовалась искусственная вентиляция легких. Однако, полученные данные не отражают, было ли это связано с неадекватной схемой антиретровирусного лечения, неудачей лечения или, возможно, причиной было существующее заболевание COVID-19. Полученные на сегодняшний день результаты проведенных исследований не поддерживают предположение об избыточной заболеваемости и высокой летальности от COVID-19 ВИЧинфицированных лиц. Как показывают наблюдения, инфекция, вызываемая вирусом новым коронавирусом SARS-CoV-2, может возникать во время применения антиретровирусной терапии [7-11].

На настоящий момент мы не имеем весомых доказательств о более тяжелом течении инфекции COVID-19 у данного контингента по сравнению с неинфицированными ВИЧ. Учеными Великобритании было проведено проспективное обсервационное когортное исследование с быстрым сбором данных и анализом практически в реальном времени с использованием предварительно утвержденного вопросника, принятого ВОЗ. В этом крупномасштабном и многоцентровом исследовании приняли участие 166 
британских больниц, 16749 пациентов с COVID-19. Средний возраст в данной выборке составлял 72 года, средняя продолжительность симптомов до поступления в лечебное учреждение - 4 дня, а средняя продолжительность пребывания в стационаре составила 7 дней.

Наиболее распространенными сопутствующими заболеваниями в исследуемой группе были хроническая болезнь сердца (29\%), сахарный диабет (19\%), хроническая обструктивная болезнь легких (19\%) и бронхиальная астма (14\%); 47\% не имели зарегистрированных сопутствующих заболеваний, только $1 \%$ в исследуемой группе составляли ЛЖВ. Увеличение возраста и сопутствующих заболеваний, включая ожирение, были связаны с более высокой вероятностью смертности, но ВИЧ не оказывал достоверного влияния на смертность таких пациентов [12].

Таким образом, наличие ВИЧ-инфекции не является предиктором тяжелого течения COVID-19, а также высокого процента летальности, что было доказано рядом исследований, проводимых в различных странах мира. Людям с ВИЧ необходимо следовать стандартным мерам профилактики новой коронавирусной инфекции (COVID-19) [13]. Пока рано говорить о том, что пациенты, живущие с ВИЧ и принимающие Калетру, защищены от коронавирусной инфекции. Так как на сегодняшний день не существует исследований, свидетельствующих о том, что данный препарат эффективен в качестве доконтактной или постконтактной профилактики при коронавирусной инфекции.

Список литературы:

1. Wuhan Municipal Health Commission briefi ng on the pneumonia epidemic situation. 31 December 2019 (in Mandarin).

2. Pneumonia of unknown cause - China. Disease outbreak news. 5 January 2020. WHO.

3. CDC. 2019 Novel Coronavirus URL https://www.cdc.gov/coronavirus/2019ncov/index.html 
4. Cao B, Wang Y, Wen D et al. A Trial of Lopinavir-Ritonavir in Adults Hospitalized with Severe Covid-19. N Engl J Med 2020; doi: 10.1056/NEJMoa2001282.

5. Kowalska J D, Skrzat-Klapaczyńska A, Bursa D, Balayan T, Begovac J, Chkhartishvili N, Gokengin D, Harxhi A, Jilich D, Jevtovic D, Kase K, Lakatos B, Matulionyte R, Mulabdic V, Nagit A, Papadopoulos A, Stefanovic M, Vassilenko A, Vasylyev M, Yancheva N, Yurin O, Horban A; ECEE Network Group. HIV care in times of the COVID-19 crisis - where are we now in Central and Eastern Europe? Int J Infect Dis. 2020 May 10. pii: S1201-9712(20)303192.

6. BHIVA, DAIG, EACS, GESIDA \& Polish Scientific AIDS Society Statement on risk of COVID-19 for people living with HIV (PLWH)

7. Blanco JL, Ambrosioni J, Garcia F, Martínez E, Soriano A, Mallolas J, Miro JM; COVID-19 in HIV Investigators. COVID-19 in patients with HIV: clinical case series. Lancet HIV. 2020 Apr 15. pii: S2352-3018(20)30111-9.

8. Härter G, Spinner CD, Roider J, Bickel M, Krznaric I, Grunwald S, Schabaz F, Gillor D, Postel N, Mueller MC, Müller M, Römer K, Schewe K, Hoffmann C. COVID-19 in people living with human immunodeficiency virus: a case series of 33 patients. Infection. 2020 May 11. doi: 10.1007/s15010-020-01438-z.

9. Guo W, Ming F, Dong Y et al. A Survey for COVID-19 among HIV/AIDS Patients in Two Districts of Wuhan, China. Preprint research paper, The Lancet, 2020.

10. Wu Q, Chen T, Zhang H. Recovery from COVID-19 in two patients with coexisted HIV infection. J Med Virol. 2020 May 13. doi: 10.1002/jmv.26006.

11. Karmen-Tuohy S, Carlucci PM, Zacharioudakis IM, Zervou FN, Rebick G, Klein E, Reich J, Jones S, Rahimian J. Outcomes among HIV-positive patients hospitalized with COVID-19. https://www.medrxiv.org/content/10.1101/2020.05.07.20094797v1.

12. Docherty AB, Harrison EM, Green CA, et al. Features of 16,749 hospitalised UK patients with COVID-19 using the ISARIC WHO clinical characterization protocol. medRxiv preprint DOI: https://doi.org/10.1101/2020.04.23.20076042.

13. Методические рекомендации MP 3.1.0170-20 «Эпидемиология и Профилактика COVID-19» Роспотребназдора. 